\title{
Modeling Method Analysis of the Friction Torque for High Speed Spindle Bearing
}

\author{
Songsheng $\mathrm{Li}^{1,2}$, HuihangChen ${ }^{1,2}$, Haibing Ding ${ }^{1,2}$, Guoye Zhang ${ }^{1,2}$ and Juan Shao ${ }^{1,2}$ \\ ${ }^{1}$ School of Mechatronic Engineering and Automation, Shanghai University, Shanghai, China, 200072 \\ ${ }^{2}$ Shanghai Key Laboratory of Intelligent Manufacturing and Robotics, Shanghai University, Shanghai, China, 200072
}

\begin{abstract}
The friction torque inside the high-speed spindle bearing determines the performance of the friction, wear, heat, temperature rise and life of the bearing. Aim to analyze the high-speed spindle bearing dynamic characteristics, considering the influence of cage and internal lubricating oil-film, a quasi-static improved model of high-speed spindle bearing was developed. And a new method for calculating the friction torque of the bearing based on the quasi-static improved model was put forward. Then the theory and experiment were validated systematically. The results show that the quasi-static improved model is correct, and the method of calculating the friction torque of high-speed spindle bearing based the quasistatic improved model is feasible and reliable.
\end{abstract}

\section{Introduction}

As important bearing parts for transmission and load, angular contact ball bearings are widely used in various high-speed rotating machines, such as high-speed spindle, Aircraft engines ${ }^{[1]}$. It is also known as the spindle bearing. Many scholars are committed to discuss appropriate analytical methods to study their dynamic characteristic and the stress state ${ }^{[2-4]}$. A. B. Jones ${ }^{[5]}$ proposed an analytical method of quasi-static. Wang Liqin, Cui $\mathrm{Li}^{[6-7]}$ et al. used the bearing part interaction model to deduce the interaction force and torque calculation process of each part. P. K. Gupta ${ }^{[8-9]}$ proposed a bearing dynamic analysis model under arbitrary operating conditions.

In the above research, simulation conditions of dynamic analysis model are too large, the calculation is too large and the calculation process is prone to divergence. Regarding quasi-dynamic analysis model, the consideration of the factors is difficult to complete and the calculation accuracy is difficult to improve. As for quasi-static model, the calculation results are very consistent with the engineering practice ${ }^{[10]}$. However, the existing quasi-static model does not take into account the role of the cage and the impact of lubricating oil film. So, the application of quasi-static analysis model has been limited. Therefore, it is necessary to take into account the impacts of factors such as cage, lubricating oil film and other factors to establish a quasi-static improved model.

Friction torque is one of the key parameters. There are two main methods for calculating the friction torque of the bearings: The first one is Palmgren's ${ }^{[11]}$ method of calculating the friction torque. It is also known as integral method; The second calculation method of friction torque proposed by Deng Si'er and Li Xinglin ${ }^{[12]}$. It is called the local method. The above two methods are difficult to combine the changes of the specific working conditions and the internal force of the bearing, it is difficult to meet the needs of actual project.

In this paper, a new method based on quasi-static improved model is proposed to analyze and calculate the friction moment of the bearing, the theoretical analysis and experimental research are carried out. It provides theoretical basis for the design, manufacture and use of high-speed spindle bearings to improve the overall performance and reliability of high-speed rotary machines.

\section{A Model of Quasi-Static Improved of Bearing}

\subsection{Geometric relationship of the bearing in the presence of oil film}

Bearing's structure is shown as figure 1. The existence of lubricating oil film will reduce the elastic deformation between the rolling element and the ring raceway ${ }^{[13]}$. Taking lubrication oil film into consideration, the position about the center of the ball and the curvature of the ring channel before and after bearing being loaded at the angular position $\phi_{j}$ is shown in figure 2 . 


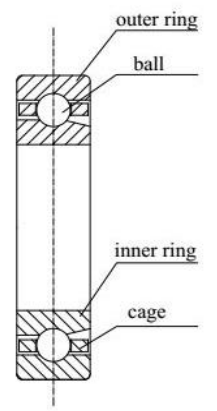

Figure 1. Structure of bearing

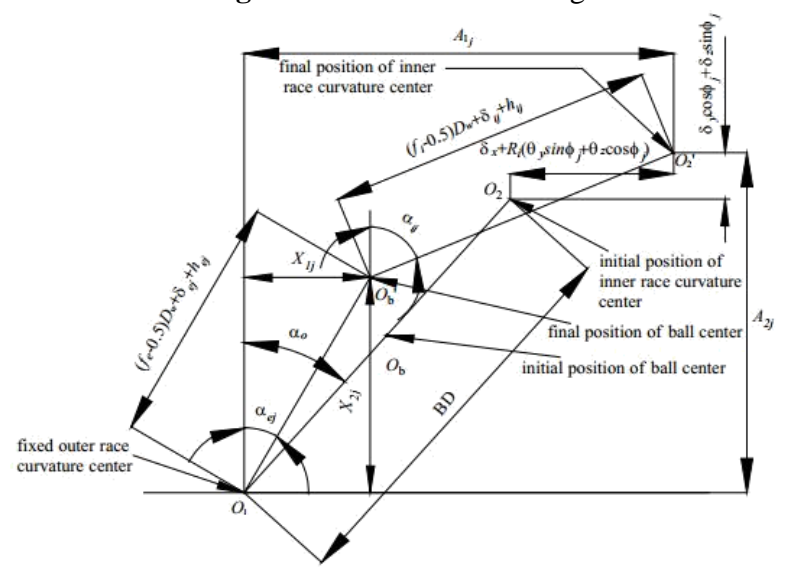

Figure 2. The positional relationship between the center of the ball and the curvature of the channel at the angular position $j$

According to the positional relationship about deformation and the geometry in the figure 1, the following equation can be obtained:

$$
\begin{aligned}
& \delta_{e j}=\sqrt{X_{1 j}^{2}+X_{2 j}^{2}}-\left(f_{e}-0.5\right) D_{w}-h_{e j} \\
& \delta_{i j}=\left\{\begin{array}{l}
\sqrt{\left(A_{1 j}-X_{1 j}\right)^{2}+\left(A_{2 j}-X_{2 j}\right)^{2}} \\
-\left(f_{i}-0.5\right) D_{w}-h_{i j}
\end{array}\right\}
\end{aligned}
$$

where, $\left(A_{1 j}, A_{2 j}\right)$ is coordinate of the inner ring groove center of curvature relative to the outer ring groove center, $\left(X_{1 j}, X_{2 j}\right)$ is the coordinate of the outer ring groove center relative to the center of the sphere, $h_{e j}, h_{i j}$ are center film thickness between the ball and the outer ring or inner ring respectively, $f_{i}, f_{e}$ are radius of ring groove, $D_{w}$ is diameter of ball.

\subsection{Ball's force analysis model}

According to the interaction about each part of the bearing, the force situation of the ball is analyzed. The state of ball suffered force is shown in figure 3, where, $M_{z g j} 、 F_{c j}$ are the gyroscopic torque and centrifugal force respectively, $Q_{i(e) j}$ is the normal contact load between the ball and the raceway, $F_{i(e) t \eta j} 、 F_{i(e) t \xi j}$ are drag forces on the contact ellipse's long and short axis respectively, $f_{R i(e) \xi j}$ is the hydrodynamic frictional force between the ball and the raceway contact inlet area, $F_{d j}$ is the viscous resistance of the oil-gas mixture to the rolling body, $F_{b c j}$, $f_{b c j}$ are the normal contact forces and tangential friction between the cage hole and the rolling body, respectively.

By analyzing force of the ball, it can conclude that the force balance equation of the ball shown as follows:

$$
\left(\begin{array}{l}
Q_{i j} \sin \alpha_{i j}-Q_{e j} \sin \alpha_{e j}- \\
F_{i t \eta j} \cos \alpha_{i j}+F_{e t \eta j} \cos \alpha_{e j}
\end{array}\right)=0
$$
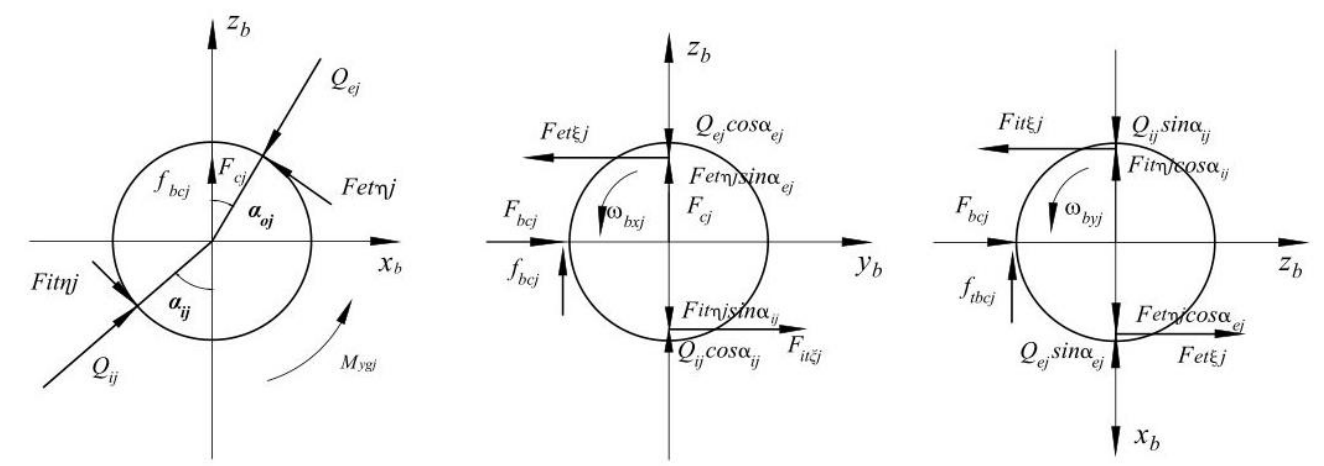

Figure 3. Force balance status of ball

$$
\begin{aligned}
& \left(\begin{array}{l}
Q_{i j} \cos \alpha_{i j}-Q_{e j} \cos \alpha_{e j}+F_{c j} \\
-F_{e t \eta j} \sin \alpha_{e j}+F_{i t \eta j} \sin \alpha_{i j}-f_{b c j}
\end{array}\right)=0 \\
& F_{e t \xi j}-F_{i t \xi j}-f_{R e \xi j}+f_{R i \xi j}+F_{b c j}-F_{d j}=0
\end{aligned}
$$

$$
\left(\begin{array}{l}
F_{e t \xi j} \frac{D_{w}}{2} \cos \alpha_{e j}+F_{i t \xi j} \frac{D_{w}}{2} \cos \alpha_{i j} \\
-f_{R e \xi j} \frac{D_{w}}{2} \cos \alpha_{e j}-f_{R i \xi j} \frac{D_{w}}{2} \cos \alpha_{i j} \\
-f_{b c j} \frac{D_{w}}{2}
\end{array}\right)=0
$$




$$
\left(\begin{array}{c}
F_{e t \xi j} \frac{D_{w}}{2} \sin \alpha_{e j}+F_{i t \xi j} \frac{D_{w}}{2} \sin \alpha_{i j} \\
-f_{R e \xi j} \frac{D_{w}}{2} \sin \alpha_{e j}-f_{R i \xi j} \frac{D_{w}}{2} \sin \alpha_{i j}
\end{array}\right)=0
$$

\subsection{Cage force analysis model}

For high-speed spindle bearings, the outer ring guides the cage generally. The movement between the ring guide surface and the cage guide surface can be seen as a special case of a journal bearings, as shown in figure 4 ,

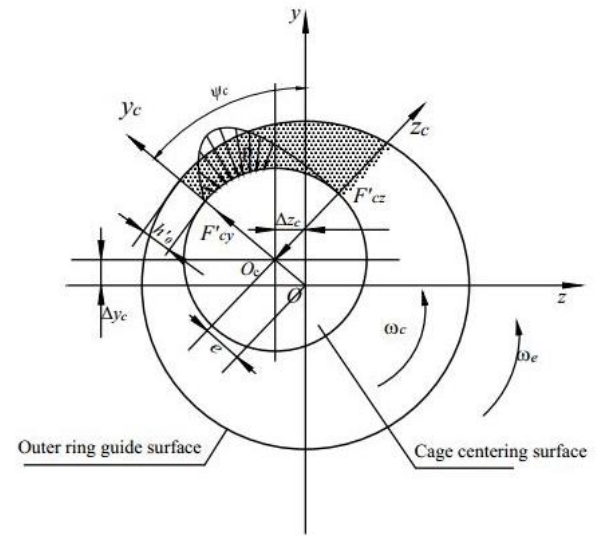

Figure 4. The interaction between Cage and outer ring

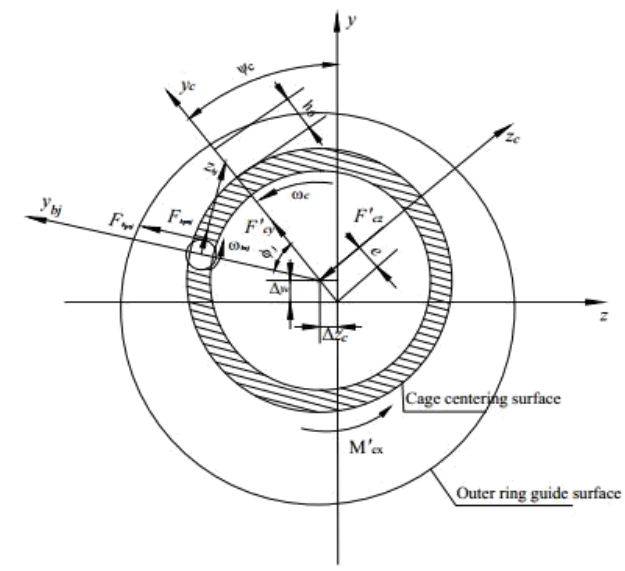

Figure 5. Force analysis of cage

where, hydrodynamic oil film force on cage is resultant force $F_{c}$. $F_{c}$ is decomposed into $F_{c y}^{\prime}, F_{c z}$. The friction torque of hydrodynamic oil film acting on cage is $M_{c x}{ }^{\prime}$.

In this paper, the cage is influenced by force of the ring and the balls, as shown in figure 5. And the force equations of cage are created, as follows:

$$
\begin{gathered}
F_{c y}-\sum_{j=1}^{Z}\left(F_{b p n j} \sin \phi_{j}-F_{b p \tau j} \cos \phi_{j}\right)=0 \\
F_{c z}-\sum_{j=1}^{Z}\left(F_{b p n j} \cos \phi_{j}+F_{b p \tau j} \sin \phi_{j}\right)=0 \\
M_{c x}-\sum_{j=1}^{Z}\left(F_{b p n j} \frac{d_{m}}{2}\right)=0
\end{gathered}
$$

\subsection{Inner ring's force analysis model}

The reaction forces of the inner ring include radial loads $F_{y} 、 \quad F_{z}$, axial loads $F_{x}$ and moment loads $M_{y} 、 M_{z}$, as shown in figure 6 . For the forces and moments acting on the inner ring to be in equilibrium as follows:
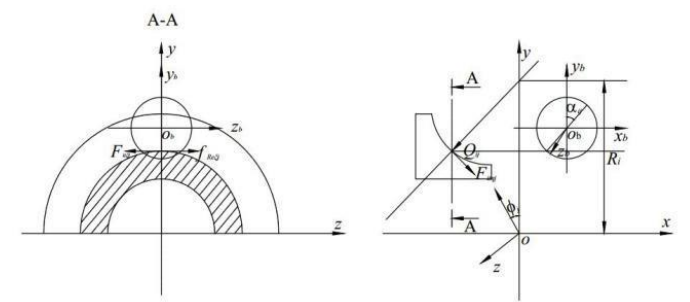

Figure 6. The interaction between the ball and the inner race

$$
\begin{aligned}
& F_{x}-\sum_{j=1}^{Z}\left(Q_{i j} \sin \alpha_{i j}+F_{i t \eta j} \cos \alpha_{i j}\right)=0 \\
& F_{y}-\sum_{j=1}^{Z}\left(Q_{i j} \cos \alpha_{i j}-F_{i t \eta j} \sin \alpha_{i j}\right) \cos \phi_{j}=0 \\
& F_{z}-\sum_{j=1}^{Z}\left(Q_{i j} \cos \alpha_{i j}-F_{i t \eta j} \sin \alpha_{i j}\right) \sin \phi_{j}=0 \\
& M_{y}-\sum_{j=1}^{Z}\left\{\begin{array}{l}
{\left[\begin{array}{l}
R_{i}\left(Q_{i j} \sin \alpha_{i j}+F_{i t \eta j} \cos \alpha_{i j}\right) \\
-f_{i} D_{w} F_{i \eta \eta j} \cos \alpha_{i j}
\end{array}\right] \sin \phi_{j}} \\
-\left[\left(F_{i t \xi j}-f_{\operatorname{Re} \xi j}\right) \sin \alpha_{i j} f_{i} D_{w}\right] \cos \phi_{j}
\end{array}\right\}=0 \\
& M_{z}-\sum_{j=1}^{Z}\left\{\begin{array}{l}
{\left[\begin{array}{l}
R_{i}\left(Q_{i j} \sin \alpha_{i j}+F_{i t \eta j} \cos \alpha_{i j}\right) \\
-f_{i} D_{w} F_{i t \eta j} \cos \alpha_{i j}
\end{array}\right] \cos \phi_{j}} \\
-\left[\left(F_{i t \xi_{j} j}-f_{\mathrm{Re} \xi_{j} j}\right) \sin \alpha_{i j} f_{i} D_{w}\right] \sin \phi_{j}
\end{array}\right\}=0
\end{aligned}
$$

Using Newton-Raphson iterative method to solving positional relationship equations, the balance equations of ball, cage stress and inner force simultaneously, force situation of all parts will be obtained finally.

\section{Model Solving and Verification}

Using fortran language to simulate and analyze and taking experimental bearing of the paper ${ }^{[14]}$ as example, the initial axial preload is $889.84 \mathrm{~N}$ and the rotating speed is $15000 \mathrm{r} / \mathrm{min}$. Through fortran language computer program, the model of quasi-static improved was calculated and simulated. Compared with the results of Jones quasi-static model and Gupta's kinetic model, the results are shown in table 1.

Table. 1 Comparison of calculation results

\begin{tabular}{|c|c|c|c|}
\hline $\begin{array}{c}\text { Bearing's } \\
\text { characteristics } \\
\text { parameters }\end{array}$ & $\begin{array}{c}\text { Jones's } \\
\text { model } \\
\text { results }\end{array}$ & $\begin{array}{c}\text { Gupta's } \\
\text { model } \\
\text { results }\end{array}$ & $\begin{array}{c}\text { Improved } \\
\text { model } \\
\text { result }\end{array}$ \\
\hline Speed (r/min) & 7255 & 7200 & 7284 \\
\hline $\begin{array}{c}\text { Inner contact } \\
\text { angle }\left(^{\circ}\right)\end{array}$ & 37.70 & 37.68 & 37.882 \\
\hline $\begin{array}{c}\text { Outer contact } \\
\text { angle }\left({ }^{\circ}\right)\end{array}$ & 4.074 & 4.015 & 4.0496 \\
\hline $\begin{array}{c}\text { Inner contact } \\
\text { load(N) }\end{array}$ & 76.49 & 76.53 & 76.522 \\
\hline $\begin{array}{c}\text { outer contact } \\
\text { load(N) }\end{array}$ & 488.44 & 477.78 & 495.29 \\
\hline
\end{tabular}


As shown in table 1, the calculation results of this improved model are basically similar to the existing quasi-static model of Jones. However, due to the effect of cage and lubricant film, the contact angle and contact load have some differences with the existing quasi-static model. The simulation results are basically consistent with results of Gupta kinetic model and the results of paper ${ }^{[14]}$. The results show that the quasi-static improved model is correct and credible.

\section{Bearing Friction Torque Model}

The friction moment is result of the friction forces of all the contact parts inside the bearing. Because the overall bearing is in a state of stress equilibrium, the role of the ball and the inner raceway reflect the role of all balls and cage and the role of all balls and the outer rings. Therefore, the friction between balls and the inner ring can reflect the bearing internal stress situation. The force of all balls acting on the inner ring multiplied by the distance from the stress point to the center axis of the bearing rotation is the friction torque, as shown in figure 7.

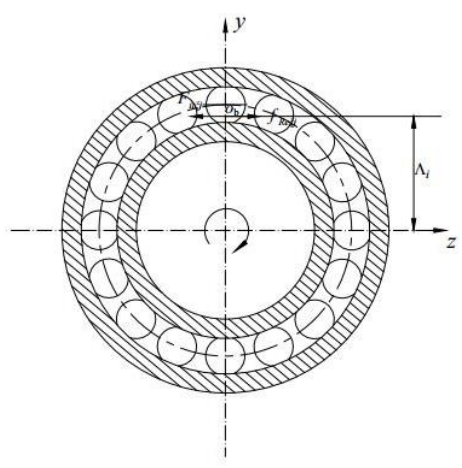

Figure 7. Schematic diagram of the ball on the role of the inner raceway

The bearing friction torque is:

$$
\begin{aligned}
M_{f} & =\sum_{i=1}^{Z}\left[\left(F_{i t \xi j}-f_{R i \xi j}\right) \Lambda_{i}\right] \\
\Lambda_{i} & =\left(\frac{1}{2} d_{m}-\frac{1}{2} D_{w} \cos \alpha_{i j}\right)
\end{aligned}
$$

where, $\Lambda_{i}$ is the distance from the stress point to the bearing center line.

Taking a manufacturer's B7006C angular contact ball bearing as an example, the influences of different working conditions on the bearing friction torque are analyzed. The bearing is lubricated with oil, the oil is 4109 aviation lubricant. Calculated results shown in figure 8 , figure 9 and figure 10 .

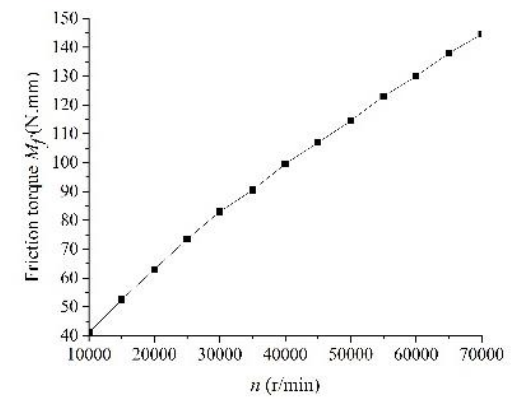

Figure 8. Influence of rotational speed on bearing friction

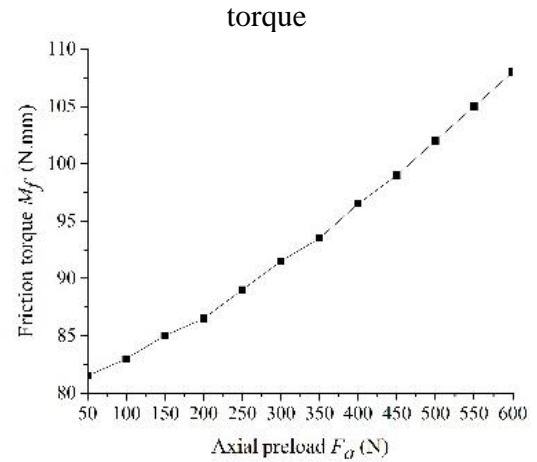

Figure 9. Influence of axial preload on bearing friction torque

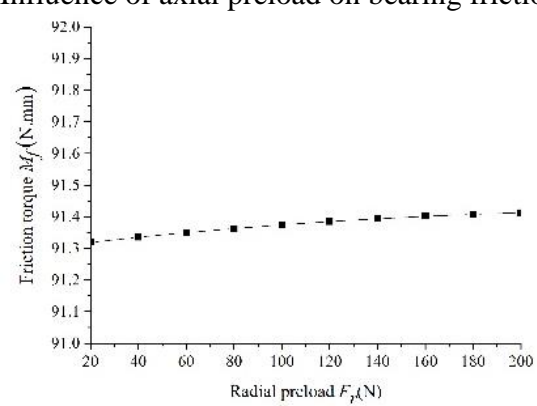

Figure 10. Influence of radial load on bearing Friction torque

As shown in figures 8 , figure 9 and figure 10 , when axial load and speed increase, the bearing friction torque increase significantly. The viscous resistance inside the pressure oil film increases, because of squeezing and shearing of the oil film, the energy consumed increases. And the radial load has a relatively small influence on the friction torque of the bearing.

\section{Related Experiments}

\subsection{Experimental Test Principle}

In this paper, the method of balancing torque ${ }^{[15-16]}$ is adopted. The friction moment test system is shown in figure 11. Device physical picture is shown in figure 12. Two sets B7006C/P4 bearings mounted on the extension end of drive spindle shaft in back to back way. The inner and outer spacers and the elastic wave ring are used to provide axial preload to the bearing. The weight provide radial preload.

A sleeve with a pressure rod on it is mounted on the outer ring of the tested bearing. When the electric spindle drives the inner race to rotate in the clockwise direction, the outer race and the sleeve will tend to rotate in the same direction due to the friction force and friction 
torque. The pressure rod on the sleeve will press against the pressure sensor fixed on the bracket. The pressure measured by pressure sensor is $F_{l} . F_{l}$ multiplied by the distance from force point to the bearing center of rotation and divided by 2 is the equivalent friction torque.

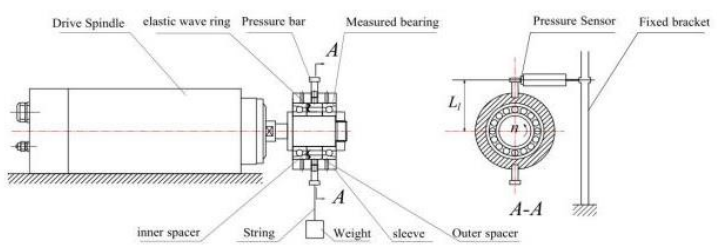

Figure 11. Bearing friction torque test system diagram

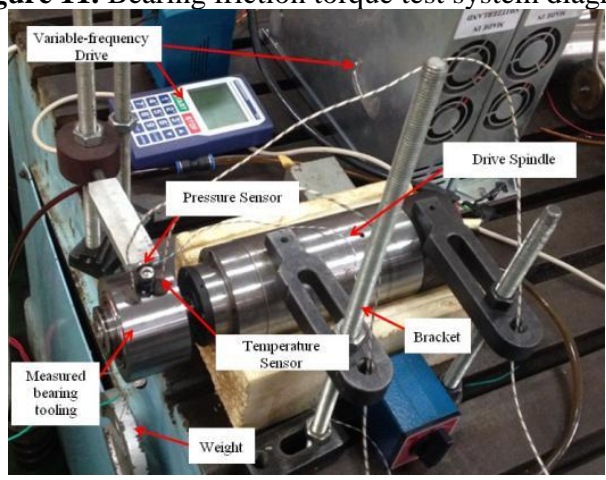

Figure 12. Bearing friction torque test device physical picture

$$
M_{f}=\frac{F_{l} L_{l}}{2}
$$

where, $M_{f}$ is rolling bearing friction torque, $F_{l}$ is measured tensile values, $L_{l}$ is distance from test point to centre of axis, the value of it is $55 \mathrm{~mm}$.

\subsection{Test results analysis}

Figure 12 (a), (b), (c), (d) and (e) show the comparison between the theoretically calculation results and the test values. The calculation results and the actual test results tend to be consistent in numerical and trends, which shows that the proposed method based on the quasi-static improved model is correct and feasible for the analysis and calculation of the bearing friction torque.

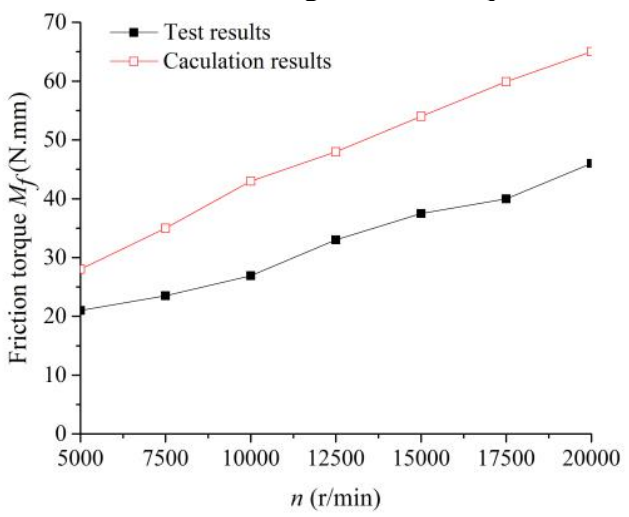

(a) $\mathrm{Fa}=130 \mathrm{~N}, \mathrm{Fr}=0 \mathrm{~N}$

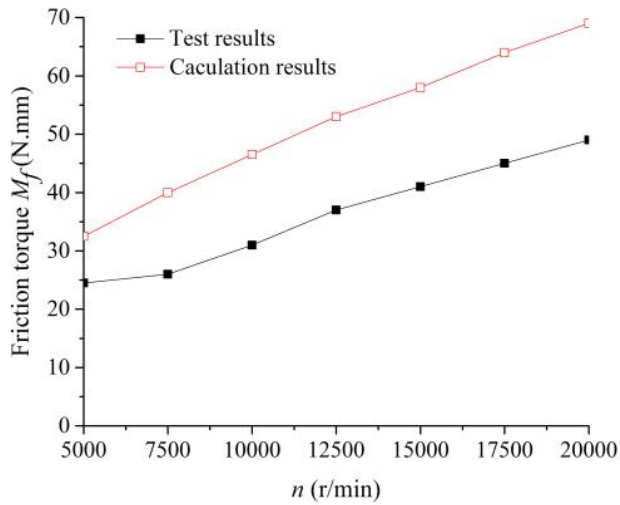

(b) $\mathrm{Fa}=240 \mathrm{~N}, \mathrm{Fr}=0 \mathrm{~N}$

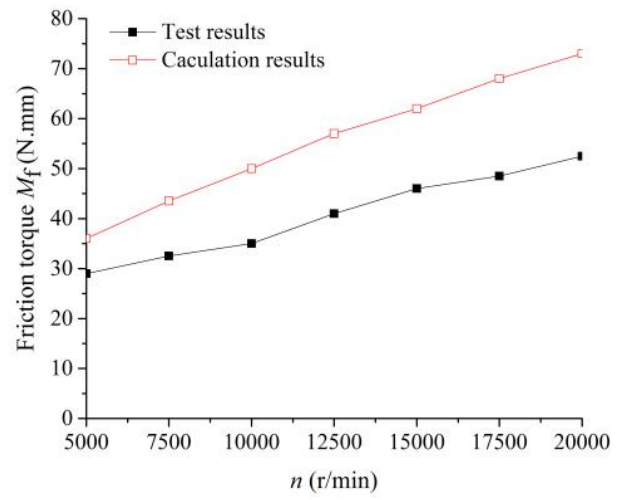

(c) $\mathrm{Fa}=320 \mathrm{~N}, \mathrm{Fr}=0 \mathrm{~N}$

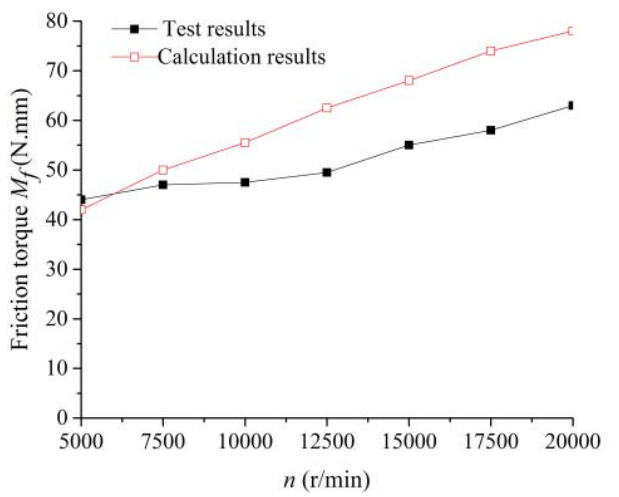

(d) $\mathrm{Fa}=430 \mathrm{~N}, \mathrm{Fr}=0 \mathrm{~N}$

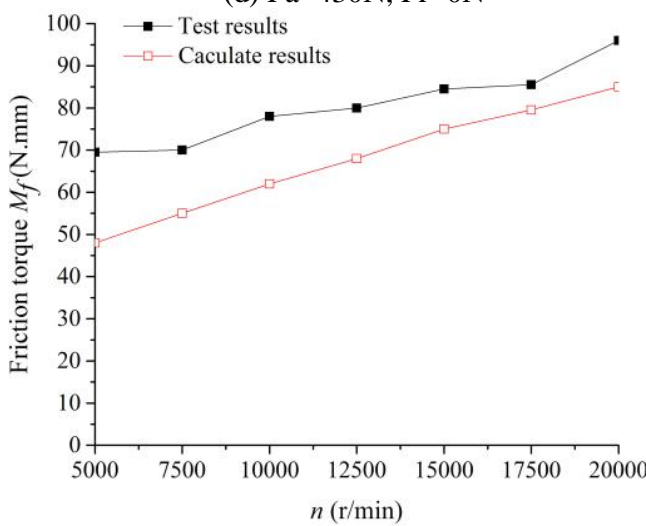

(e) $\mathrm{Fa}=530 \mathrm{~N}, \mathrm{Fr}=0 \mathrm{~N}$

Figure 12. Comparison of friction torque test and theoretical calculation results

\section{Conclusion}

(1) Under the condition of high-speed running, the role of cage and the impact of the oil film inside the bearing 
cannot be ignored, the quasi-static model cannot analyze friction torques and other important parameters, so that its usage is limited.

(2) Based on the quasi-static analysis method, considering the effect of the cage and the influence of lubricating oil film inside the bearing, the quasi-static improved model is more correct and more in line with the actual engineering.

(3) Based on the quasi-static improved model, the proposed method of analyzing the bearing frictional moment is correct and feasible, which is more in line with engineering practice.

\section{References}

1. G Chen. Aeroengine structure design analysis. (Beijing University of Aeronautics and Astronautics Press, 2006)

2. S S Li, L X Yang, G Zhang, et al. B, 4, 34 (2005)

3. W L Xiong, F F Li, Z H Ji, et al. TMMT, 3, 25 (2010)

4. C S Wan. Analysis of rolling bearings. (Mechanical Industry Press, 1985)

5. Jones A B. ASME, 82, 309 (1960)

6. L Cui, L M Wang, D Z Zheng, et al. AAAS, 29, 492 (2008)

7. L Q Wang, L Cui, D Z Zhang, et al. AAAS, 28, 1461 (2007)

8. Gupta P K. ASME, 101, 312 (1979)

9. Gupta P K. ASLE, 26, 293 (1983)

10. J Shao. Modeling method analysis and dynamic characteristics of high-speed spindle bearing. (Shanghai University, 2015)

11. Palmgren A. Ball and roller bearing engineering. (3rded PhiladelPhia: Burbank, 1959)

12. S E Deng, L X Li, J G Wang, et al. JME, 3, 114 (2011)

13. S S Li, J Shao, F Yu, et al. AMR, 97, 945 (2014)

14. Gupta P K. JLT, 97, 261 (1975)

15. X K Gao, D A Wan. SHMM, 6, 13 (2003)

16. L Wei, S G Zhang, F X Duan, et al. B, 3, 61 (2013) 